\title{
Multidisciplinary Breast Conference Improves Patient Management and Treatment
}

\author{
Vijayashree Murthy*, Silvana Nobre, Lauren Sparber, Sarah Schaefer, Elissa Santoro, \\ Janette McDermott, Ronald Chamberlain, Michele Blackwood \\ Saint Barnabas Medical Center, Livingston, USA \\ Email: ㅁvijumurthy@gmail.com
}

Received 22 May 2014; revised 18 June 2014; accepted 14 July 2014

Copyright (C) 2014 by authors and Scientific Research Publishing Inc.

This work is licensed under the Creative Commons Attribution International License (CC BY).

http://creativecommons.org/licenses/by/4.0/

c) (i) Open Access

\begin{abstract}
Introduction: Multidisciplinary breast conference (BC) that discusses different clinicopathological aspects of disease creates a sound, complete and unique treatment plan for each patient. The aim of this study was to investigate the role of weekly breast tumor boards in patient management and treatment modifications. Methods: From June 2010 to June 2011, 242 patients (Stage 0 to Stage 4 breast cancer) were prospectively presented before a multidisciplinary team. Presentation included clinical data, imaging and pathological review followed by prospective treatment plan formulated by the physician group. If postoperative presentation, final histopathology of the tumor and adjuvant treatment relevant to each patient was discussed. Breast conference was performed via teleconference, including video projection of pathology slides and images. Feedback evaluations were completed and returned to the Tumor Registry for consistent weekly improvement of BC. Results: 102 patients (42\%) had modified management after being presented at $\mathrm{BC}$, $38 \%(n=39)$ surgical, $33 \%(n=34)$ medical (chemotherapy/endocrine therapy), $17 \%(n=17)$ radiation, $6 \%(\mathrm{n}=6)$ both medical and radiation treatment and $6 \%(\mathrm{n}=6)$ imaging changes. Majority of changes were made to patients with Stage IA (27\%) followed by Stage $0(21 \%)$ breast cancer. Conclusion: Breast conference presents a significant impact on patient management and serves as a model for best practice in cancer care. Consultative discussion of each case permits every team member to be acutely aware of the patient's condition, contribute to treatment plan and permit a harmonious and cooperative team approach while assuring best treatment for the patient.
\end{abstract}

\section{Keywords}

Breast Conference, Multidisciplinary Breast Meeting, Breast Tumor Board

\footnotetext{
${ }^{*}$ Corresponding author.
}

How to cite this paper: Murthy, V., Nobre, S., Sparber, L., Schaefer, S., Santoro, E., McDermott, J., Chamberlain, R. and Blackwood, M. (2014) Multidisciplinary Breast Conference Improves Patient Management and Treatment. Surgical Science, 5, 314-319. http://dx.doi.org/10.4236/ss.2014.57053 


\section{Introduction}

Breast cancer remains the most common cancer accounting for $>25 \%$ of all malignancies diagnosed in US women [1]. 226,870 new cases of breast cancer are estimated nationally in 2012, and with over 39,510 patients likely to die of breast cancer [1]. The standard of care for breast carcinoma has evolved over recent decades from radical local surgical treatment such as mastectomies, to include complex and interdisciplinary treatment. As contemporary care has developed into the standard, other aspects of treatment have also expanded. Newer developments in medical and radiation oncology have become integral to breast cancer treatment. Management of most patients is straightforward, however some patients present diagnostic or treatment dilemmas with respect to their age, family history, genetics, presentation or tumor biology. In our march towards personalized medicine and treatment or, given the fact that some patients may present uniquely, standard guidelines of the National Cancer Comprehensive Network (NCCN) may be difficult to implement in some situations. As such, a more robust multidisciplinary approach with the cooperative efforts of breast and reconstructive surgeons, medical and radiation oncologists, pathologists, radiologists and other medical professionals is required. A multidisciplinary breast conference that discusses the different clinicopathological aspects of the disease creates a sound, complete and unique treatment plan for each patient. As per the National Accreditation Program for Breast Centers, centers with more than 250 new cases of breast cancer each year are required to have a weekly breast conference [2]. Centers who have less than 250 new cases per year are required to have breast conference "every two weeks or twice monthly" [2]. Despite these recommendations, few published studies have focused on the benefits of a multidisciplinary breast tumor board and its ability to impact patient management. The aim of this study is to investigate the role of weekly breast tumor boards on patient management and how they led to treatment modifications.

\section{Methods}

Saint Barnabas Medical Center (SBMC), Livingston, NJ sees approximately 10,500 abnormal mammograms and approximately 500 new cases of breast cancer annually.

After approval by the Institutional Review Board (IRB \#11-54), 242 breast cancer patients presenting to SBMC between June 2010 and June 2011 who were presented before a multidisciplinary team weekly were analyzed (average 6 cases/conference). The multidisciplinary team consisted of breast surgeons, plastic surgeons, radiation and medical oncologists, radiologists, pathologists, residents, medical students and nurses. Demographic data including age, family history of breast and/or ovarian cancer, genetic test positivity and clinical presentation along with relevant breast imaging and fine needle aspiration cytology (FNAC)/biopsy results were presented and a prospective plan for management (therapeutic-surgery or neoadjuvant chemotherapy followed by appropriate adjuvant treatment or palliative-chemotherapy and/or hormone/targeted therapy) was developed. Given SBMC’s multiple campuses, breast conference was performed via teleconference, which included video projection of pathology slides and images. Once a month, the entire team met together in a common conference room. The format of breast conference was as follows: presenting physician introduced the case, beginning with chief complaint, physical exam, surgery performed, pathology and initial recommendation. The radiology department then presented any available images and presumed clinical diagnosis imaging. Pathologists reviewed slides received from surgical procedures (biopsy, lumpectomy, etc.) and confirmed diagnosis. Following these initial presentations, questions concerning the patients were addressed and a conclusive treatment plan was derived. The need for adjuvant therapy as well as any additional surgical procedures was carefully considered.

Feedback evaluations were distributed, completed and returned to the Tumor Registry for consistent weekly improvement of breast conference. Each of the 242 cases was analyzed for prospective treatment plan, genetic testing, type of surgery, and whether changes were made to the treatment plan after presentation at breast conference. If breast conference provided a change to treatment plan, "yes” was applied to the patient case. Changes were categorized into surgical, medical, radiation, radiological or combined medical and radiation (Table 1 and Table 2). Changes in patient management were totaled individually by presenting physician and then overall cases. Percentages were calculated for both categories. Patients who received a pathologic staging were also totaled, and changes to these patients' treatment were analyzed and categorized by presenting physician and overall cases. Demographic and clinicopathological data was collected for all 242 patients and student $\mathrm{T}$ test and Chi square tests were performed using Statistical Predictive Software and Solutions (SPSS) ${ }^{\mathrm{TM}}$ for continuous and categorical variables, to evaluate type of diagnostic or treatment changes based on specific patient and tumor factors. 
Table 1. Specific types of change incorporated into patient treatment plans after discussion at a multidisciplinary breast conference.

- Surgical change-lumpectomy to mastectomy or vice versa, breast reconstruction, additional resection of surgical margins, need for additional dissection of sentinel lymph nodes or axillary dissection.

- Medical change-addition or removal of chemotherapy, or chemotherapy dose or endocrine therapy, recommendation for Oncotype $\mathrm{DX}^{\circledR}$ testing.

- Radiation change_-addition or removal of radiation therapy, type of portals.

- Radiologic change-additional mammogram, ultrasound or MRI testing.

- Combined medical and radiation change-addition or removal of chemotherapy, endocrine therapy as well as radiation therapy.

Table 2. Changes to treatment of staged patients as a percentage of total patients presented at breast conference.

\begin{tabular}{|c|c|c|c|c|c|c|c|}
\hline Physician & $\begin{array}{l}\text { Number of } \\
\text { presented } \\
\text { cases in breast } \\
\text { conference } \\
(\mathrm{n}=242)\end{array}$ & $\begin{array}{l}\text { Total number } \\
\text { of cases with } \\
\text { change in plan }\end{array}$ & $\begin{array}{l}\text { Change in } \\
\text { surgical } \\
\text { treatment }\end{array}$ & $\begin{array}{l}\text { Change in } \\
\text { medical } \\
\text { treatment }\end{array}$ & $\begin{array}{l}\text { Change in } \\
\text { radiation } \\
\text { treatment }\end{array}$ & $\begin{array}{l}\text { Change in } \\
\text { combined } \\
\text { medical and } \\
\text { radiation } \\
\text { treatment }\end{array}$ & $\begin{array}{l}\text { Change in } \\
\text { radiological } \\
\text { management }\end{array}$ \\
\hline $\begin{array}{c}\text { Physician A } \\
\text { (surgeon) }\end{array}$ & 139 & 66 (47\%) & 20 (30\%) & 29 (44\%) & 11 (17\%) & $4(6 \%)$ & $2(3 \%)$ \\
\hline $\begin{array}{l}\text { Physician B } \\
\text { (surgeon) }\end{array}$ & 46 & 15 (33\%) & 10 (67\%) & $1(7 \%)$ & $1(7 \%)$ & $1(7 \%)$ & $2(13 \%)$ \\
\hline $\begin{array}{c}\text { Physician C } \\
\text { (surgeon) }\end{array}$ & 6 & $4(67 \%)$ & 3 (75\%) & $1(25 \%)$ & - & - & - \\
\hline $\begin{array}{l}\text { Physician D } \\
\text { (surgeon) }\end{array}$ & 32 & 8 (28\%) & 2 (25\%) & 2 (25\%) & 3 (35\%) & 1 (13\%) & - \\
\hline $\begin{array}{l}\text { Physician E } \\
\text { (medical } \\
\text { oncologist ) }\end{array}$ & 5 & $2(40 \%)$ & - & $1(50 \%)$ & $1(50 \%)$ & - & - \\
\hline $\begin{array}{l}\text { Physician F } \\
\text { (medical } \\
\text { oncologist) }\end{array}$ & 14 & 7 (50\%) & $4(57 \%)$ & - & 1 (14\%) & 1 (14\%) & $1(14 \%)$ \\
\hline
\end{tabular}

\section{Results}

242 cases were presented before the multidisciplinary team during the 12-month period analyzed (June 2010June 2011). 102 patients (42\%) had management modified as a result of breast conference presentation. Among this group 38.2\% ( $\mathrm{n}=39$ cases) resulted in changes in surgical treatment, 33.3\% ( $\mathrm{n}=34$ cases) medical management (chemotherapy/endocrine therapy), $16.6 \%(n=17$ cases) radiation treatment, $6.8 \%(n=7$ cases) combined medical and radiation therapy and $4.9 \%(\mathrm{n}=5$ cases) imaging changes (MRI, mammogram, PET CT, etc.) (see Figure 1). Among the 242 patients, 194 received a staging by pathology. 84 of these patients (43\%) received a change in treatment after presentation at breast conference. The majority of changes made after presentation at tumor board were in patients with Stage IA disease (27\%), followed by patients with Stage 0 disease (21\%) (see Figure 2).

Six different physicians presented the 242 cases included in this study. Physician A presented 139 cases, Physician B, 46; Physician C, 6; Physician D, 32; Physician E, 5; Physician F, 14. Physicians A-D were surgeons while Physicians E and F were oncologists. Physician A's case presentations resulted in changes in patient management and treatment in sixty-six (47\%) cases. Twenty patients (30\%) received surgical treatment changes, 29 patients (44\%) received changes to medical treatment, 11 patients $(17 \%)$ received changes to radiation treatment, 4 patients (6\%) received changes to both medical and radiation treatment and two cases (3\%) resulted in imaging changes. 128 (92\%) of Physician A's patients were given a pathologic staging and fifty-six (44\%) of these patients had their management changed by breast conference. The majority of changes suggested involved patients with Stage IA disease (26\%), closely followed by patients with Stage IIB disease (17\%).

Physician B's case presentations resulted in fifteen treatment changes (33\%). Of these fifteen changes, ten were surgical (67\%), one was medical (7\%), one was radiation (7\%), one was both medical and radiation (7\%) and two were radiologic (13\%). Physician B's patients were also given clinical staging; 33 of 46 patients (72\%) were staged. Thirteen (40\%) staged patients received changes to treatment after presentation at breast conference. The majority of changes suggested involved patients with Stage 0 and Stage IIA disease, each with a 25\% 


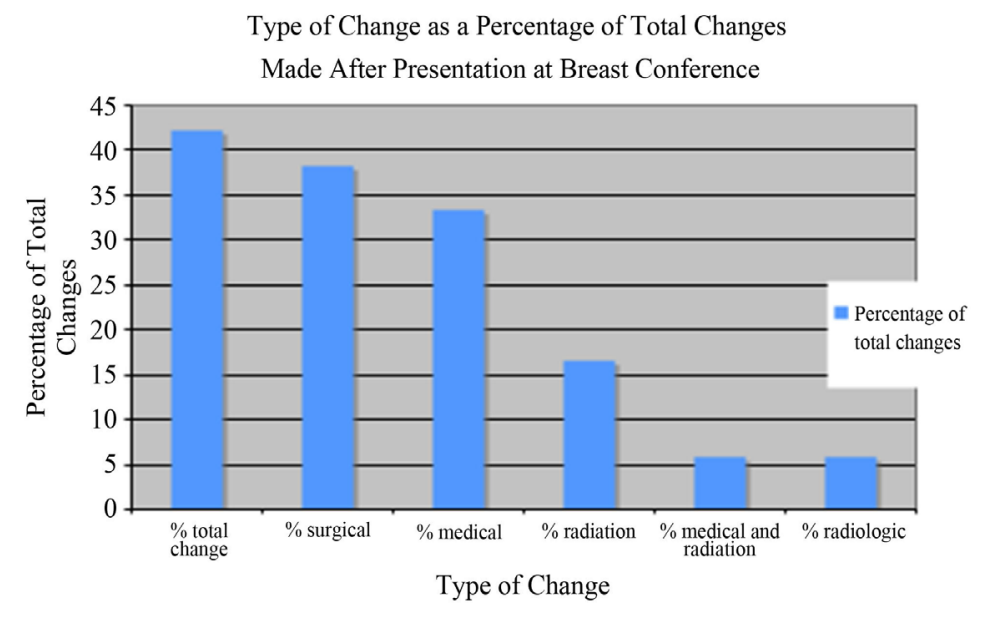

Figure 1. Type of treatment modification as a percentage of total change made following discussion at breast conference. Total $42 \%$ of all cases presented received changes to treatment following discussion at breast conference $(38.2 \%$ surgical, $33.3 \%$ medical, $16.6 \%$ radiation, $6.8 \%$ both medical and radiation and $4.9 \%$ radiologic).

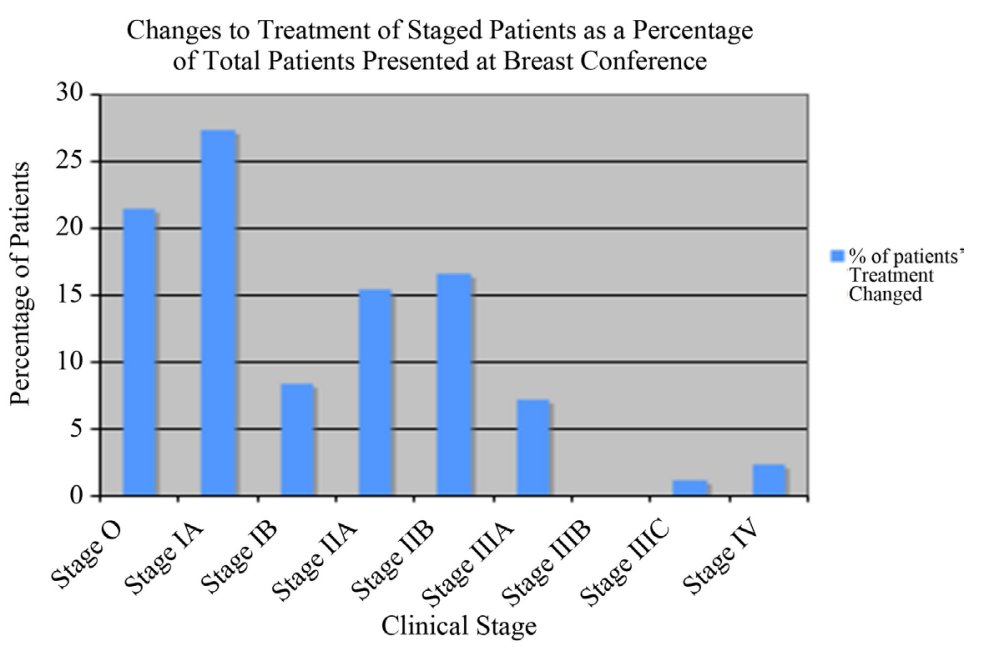

Figure 2. Changes to treatment of staged patients as a percentage of total patients presented at breast conference. Changes in Stage 0 patient treatment total $21 \%$ of total changes. Stage IA: $27 \%$; Stage IB: $8 \%$; Stage IIA: $15 \%$; Stage IIB: $17 \%$; Stage IIIA: 7\%; Stage IIIB: 0\%; Stage IIIC: 1\%; Stage IV: $2 \%$.

change, closely followed by patients with Stage IA and IIB disease, each with $12.5 \%$ change.

Physician C presented six cases, four (67\%) of which resulted in suggested treatment changes. Three patients (75\%) received recommendation for surgical treatment change and one patient (25\%) received a medical treatment change recommendation. While all six patients were staged, four treatment plans were changed due to breast conference recommendations. The majority of changes made involved patients with Stage IA disease (50\%).

Eight cases (28\%) presented by Physician D received suggested changes to the treatment plan after breast conference discussion. Two cases (25\%) resulted in surgical changes, two cases (25\%) received medical treatment change recommendations, three cases (38\%) received radiation treatment change recommendations and one case (13\%) received a change in radiological investigation recommendation. Twenty-eight of 32 patients (88\%) were given pathologic staging. Seven (25\%) of these patients' treatment was modified after presentation at breast conference. The majority of changes suggested involved patients with Stage 0 disease (50\%), followed by patients with Stage IA disease (25\%).

Physician E's presentations resulted in recommendations for treatment change in two patients (40\%), equally 
divided between medical and radiation changes (50\% each). Physician E's patient information did not include pathologic staging.

Physician F's presentations resulted in recommendation for change in treatment for seven patients (50\%). Four cases (57\%) resulted in surgical changes, while one case (14\%) resulted in radiation treatment changes, one case $(14 \%)$ resulted in both medical and radiation treatment changes and the last case (14\%) resulted in radiologic changes. Eight of Physician F's patients were given a pathologic staging. Among staged patients, three cases resulted in treatment changes, equally distributed at $14 \%$ between Stage 0, IIB and IV.

Each patient's surgery was recorded throughout the study period. Fifty-nine percent of patients in this study received breast conservation therapy, while $35 \%$ received mastectomies. The remaining $5 \%$ received other procedures (core biopsies, chest wall excisions, etc) or no surgery at all (see Figure 3). Seventy-three percent of patients who underwent mastectomies opted for reconstructive surgery.

Genetic testing was also enumerated throughout our study. Fifty-three patients (29\%) were tested for the BRCA genes. Eighty-five percent of those patients tested negative for both BRCA1 and BRCA2. Of the remaining 15\%, 75\% tested positive for BRCA2 and 25\% were positive for BRCA1.

\section{Discussion}

Cancer treatment can be complex and confusing to both physicians and patients in specific unique situations. Increasing specialization and complexity of knowledge has led to the need for multi-disciplinary teams (MDTs) to guide the management of patients with cancer. The National Cancer Comprehensive Network Tumor review boards are used in a variety of oncology settings; and have been shown to improve patient care [3]. Breast oncology is no exception. An interdisciplinary breast conference has been a longstanding requirement by the National Accreditation Program for Breast Centers. This integral aspect of breast cancer care not only aligns patient care between specialties but has also been shown to result in changes to patient management and treatment plans. Controversial or ambiguous cases are when breast conferences are perhaps most important in guiding patient management. Table 3 discusses the benefits and pitfalls of multidisciplinary care and research for breast cancer [4]. With a team of expert physicians, certain National Comprehensive Cancer Network (NCCN) guidelines can be negotiated when other factors are taken into consideration, for example, patient co-morbidities, age, etc. Breast cancer teams also permit team members to keep up to date with current literature and integrate this new literature into patient care while also promoting the most beneficial treatment plan. Of note certain issues around multidisciplinary conferences pose a dilemma that needs to be clarified:

- Approaches to patient consent before a multidisciplinary meeting.

- Professional liability implications of team-based decisions compared with individual practitioner recommendations.

- Documentation of meeting outcomes.

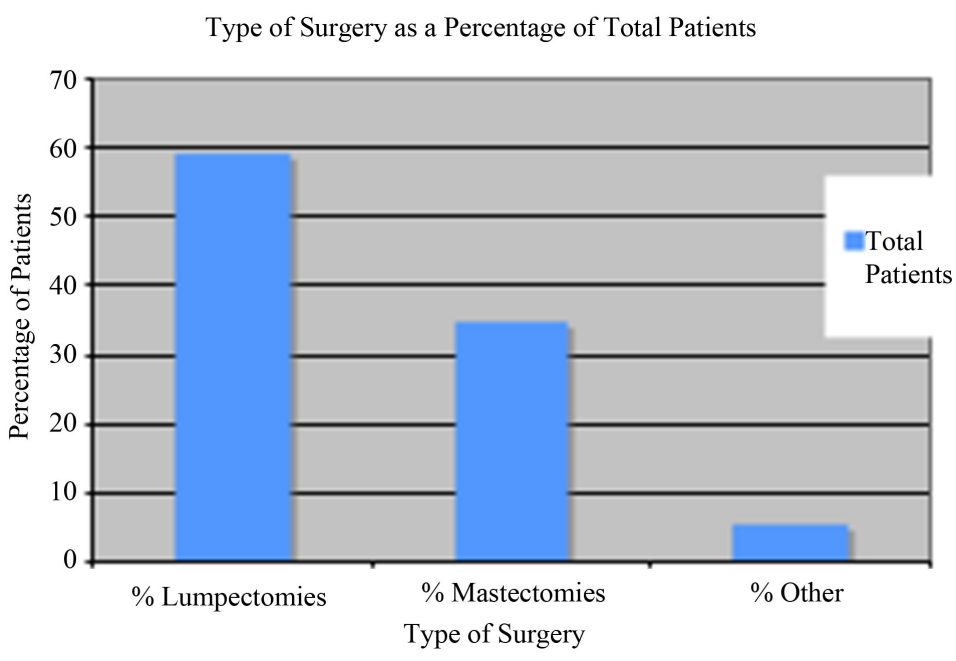

Figure 3. Type of surgery as a percentage of total patients. 59\% of patients received lumpectomies; $40 \%$, mastectomies and $5 \%$ other or no surgery. 
Table 3. Benefits and pitfalls of multidisciplinary care and research for breast cancer.

\begin{tabular}{|c|c|}
\hline \multicolumn{2}{|l|}{ Clinical care } \\
\hline Benefits & Pitfalls \\
\hline Single site of care & Incompatible space/personnel needs \\
\hline Decreased cost (depends on volume) & Increased cost (depends on volume) \\
\hline Shared resources (charts, staff, equipment) & Increased complexity of staff function/training \\
\hline Better communication & Turf wars, politics \\
\hline Coordinated treatment plan & \\
\hline Broad based patient education & \\
\hline Coordinated follow up & \\
\hline \multicolumn{2}{|l|}{ Research } \\
\hline Pooled resources (biostatistical informatics, equipment/reagents, staff, space) & Increased complexities and incompatabilities \\
\hline Correlations and input across specialities & Scientific disagreements \\
\hline Enhanced development of evidence for practice and further research & Different "cultures" and educational backgrounds \\
\hline Cross training/education across disciplines & Politics of different departments and research units \\
\hline
\end{tabular}

The recommended treatment plan should be documented and included in the patient record [5]. If more than one option is recommended, or if there are dissenting views about the course of treatment to be recommended, this should be documented in the treatment plan and communicated to the patient. The recommended treatment plan should not be implemented until the patient has agreed to a course of action and after appropriate counseling that complies with contemporary standards of patient disclosure.

\section{Conclusions}

Multidisciplinary breast conference had a significant impact on breast cancer patient management and treatment, resulting in change of care for $42 \%$ of presented cases. Consultative discussion of each patient case permits all members of the breast cancer team to contribute to the development of an individual treatment plan. Group presentation also allows all treating physicians the opportunity to be aware of patient's unique situations and improve dialogue and cooperation among members of the breast cancer team.

In this modern era of personalized medicine and treatment, a weekly breast conference designed to discuss modification of diagnostic, medical, surgical and radiation treatment plans, especially in ambiguous cases, inclusion of validated genetic and hormonal factors, discussion of targeted therapy when appropriate and patient accrual into clinical trials, permits the development of optimal individual treatment protocols for each patient. Important issues which need to be further addressed in regards to multidisciplinary breast conference includes patient consent for discussion and professional liability implications of team-based decisions compared with individual practitioner recommendations, particularly when recommendations deviate from established standards.

\section{References}

[1] American Cancer Society (2012) Cancer Facts and Figures. http://www.cancer.org/acs/groups/content/@epidemiologysurveilance/documents/document/acspc-031941.pdf

[2] American College of Surgeons (2011) Accreditation. http://napbc-breast.org/accreditation/accreditation.html

[3] Nguyen, N.P., Vos, P., Lee, H., Borok, T.L., Karlsson, U., Martinez, T., Welsh, J., Cohen, D., Hamilton, R., Nguyen, N., Nguyen, L.M. and Vinh-Hung, V. (2008) Impact of Tumor Board Recommendations on Treatment Outcome of Locally Advanced Head and Neck Cancer. Oncology, 75, 186-191. http://dx.doi.org/10.1159/000163058

[4] Tripathy, D. (2003) Multidiscplinary Care for Breast Cancer: Barriers and Solutions. Breast Journal, 9, 60-63. http://dx.doi.org/10.1046/j.1524-4741.2003.09118.x

[5] Evans, A.C., Zorbas, H.M., Keaney, M.A., et al. (2008) Medicolegal Implications of a Multidisciplinary Approach to Cancer Care: Consensus Recommendations from a National Workshop. Medical Journal of Australia, 188, 401-404. 
Scientific Research Publishing (SCIRP) is one of the largest Open Access journal publishers. It is currently publishing more than 200 open access, online, peer-reviewed journals covering a wide range of academic disciplines. SCIRP serves the worldwide academic communities and contributes to the progress and application of science with its publication.

Other selected journals from SCIRP are listed as below. Submit your manuscript to us via either submit@scirp.org or Online Submission Portal.
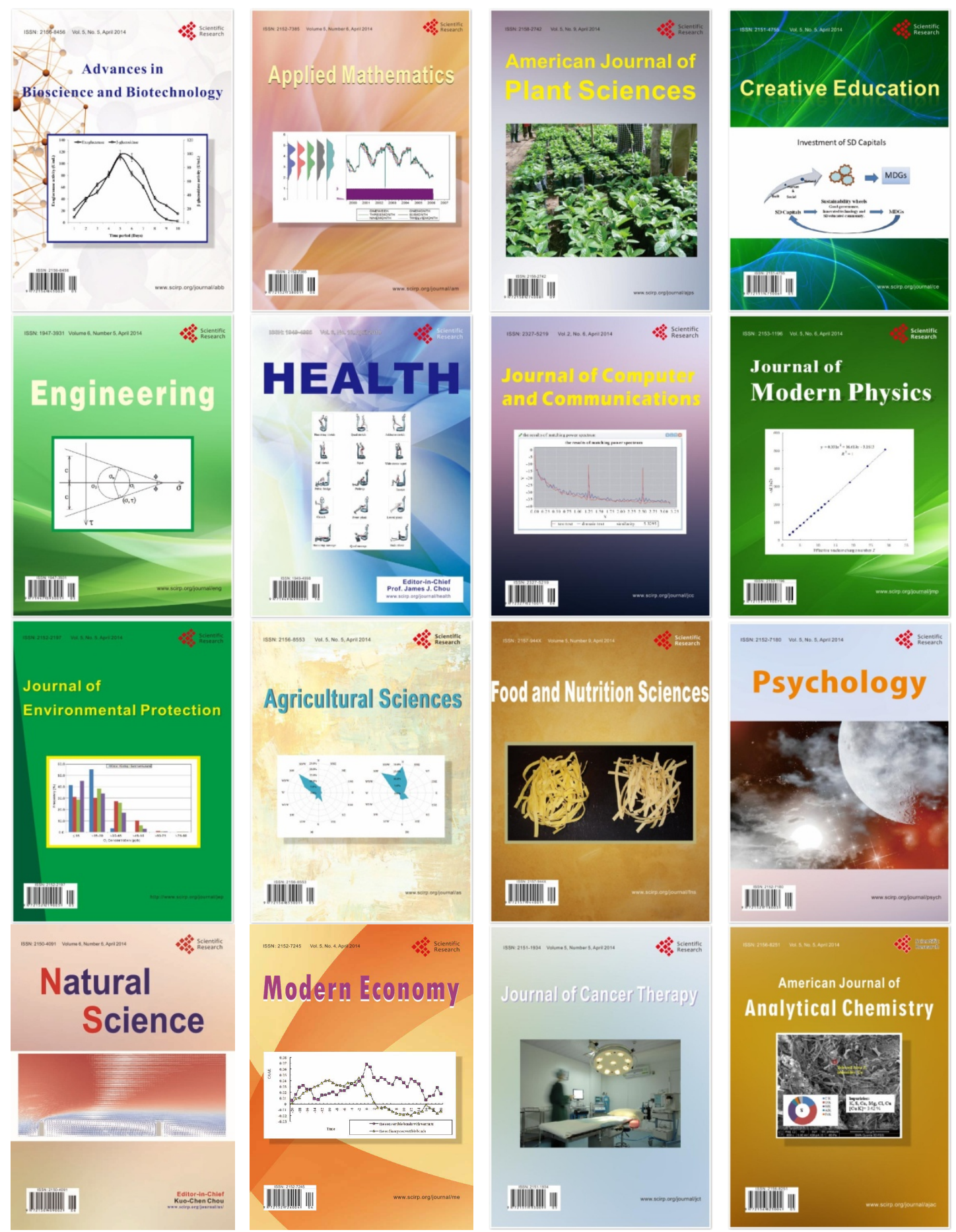